recent observation by Griffiths (Science 201,$525 ; 1978$ ) that electron micrographs of $\varnothing \times 174$ DNA suggest a nucleotide repeat of only $2.9 \AA$, instead of the $3.4 \AA$ expected for $\boldsymbol{B}$-DNA. The alternative explanation preferred by some workers is the existence in solution of a $B$-like structure with slightly more than 10 residues per turn (Wang Proc. natn. Acad. Sci. U.S.A. 76, 200; 1979).

The authors finish with a flourish of speculation about the possibility of travelling disturbances. The idea that DNA can 'breathe' and develop a travelling region of denaturation is an old one, and the idea of travelling kinks has already been mentioned; it now seems possible that travelling discontinuities between regions of different DNA conformation might be important in biological recognition. The sequence-specific conformational preferences that have been shown for DNA would, they say, act as 'sources, sinks and barriers' for transient conformational discontinuities in the helix. The idea of DNA as an inert repository of genetic information seems really to have breathed its last.

\section{Locust phase colouration}

\section{from Richard J. Moore}

Change in colouration is the most noticeable feature of the 'phase' transformation which locusts undergo during their large population fluctuations, yet its adaptive significance has proved one of the most difficult to explain. Individuals in low population densities during plague recessions (solitaria phase) are a uniform green, presumably a disguise from the attentions of visually-guided predators. During plague outbreaks, individuals develop a striking black and yellow or orange colouration (gregaria phase).

This suggests an aposematie or warning function to predators; yet locusts are voraciously pursued by flocks of birds, and nomads regard them as something of a delicacy. Batesian mimicry of a poisonous or distasteful model is unlikely to be operating since no possible models with a similar range are known, and in any case the vast numbers of locusts in swarms would greatly outnumber any model.

Early research on the problem focused on the possible role of the gregaria colour pattern in maintaining migratory groups. In 1928, Boris Uvarov suggested that the increased activity of hoppers (nymphs) in bands resulted from an optomotor or visual compensation response to

Richard Moore is a member of the Cambridge C.S. Research Group, a contract research organisation specialising in tropical marine conservation. movement of neighbours. Peggy Ellis ( $J$. exp. Biol. 30, 214; 1953) found, however, that a background of moving vertical stripes was not as effective in maintaining the characteristic 'marching' of hoppers as visual stimuli from moving locusts. This and a more recent experiment by Sylvia Gillett suggests that locust shape is the more important stimulus for grouping. Gillett (Anim. Behav. 21, 153; 1973) mixed normally-pigmented gregaria desert locusts with albinos, which aggregate less than coloured individuals. Mixing them neither reduced the grouping of normal locusts, nor increased the grouping of albinos: thus the reduced grouping of albinos alone must be due to some other factor besides lack of colouring.

It should be noted that Gillett's experiment examined static aggregation; and also that the vertical stripes in Ellis's experiment were not specifically designed to imitate gregaria locust patterning. So it remains a possibility that the patterning has evolved to maximise the optomotor response, and thus cohesion of moving groups.

Recently, however, workers have begun to examine the role of predation as the evolutionary force promoting phase polymorphism; indeed, aggregation itself may give a defensive advantage against predators. Matthews proposes (Amer. Nat. 111, 213; 1977) that individual locusts, and also Australian stick insects (which undergo remarkably similar density-dependent phase transformations), contain small amounts of toxins, so that the consumption of large numbers of them by a predator would eventually exceed its tolerance limit. Remembrance of the strikingly-coloured prey which had produced the intoxication would result in a specific avoidance image (a negative 'search image') preventing further consumption of locusts. It is doubtful, though, how much protection would be conferred in this way, since large birds such as storks are known to be capable of consuming in the order of 1000 adult locusts per day, day after day.

Gillett has begun to look at the factors affecting locusts' vulnerability to predation. Against a green irregular background, surprisingly, gregaria nymphs are just as effectively camouflaged as solitaria (Gillett \& Gonta Anim. Behav, 26,$282 ; 1978$ ). But in a strategy of crypsis, density is also of major importance, as her experiments showed. In the wild, gregaria nymphs often occur massed in numbers so great as to preempt any possibility of escaping detection. These very numbers, and their increased activity, might make it difficult for a predator to pick off individual locusts. Could the gregaria pattern contribute to this?

In her latest work (Gillett, Hogarth \& Noble, Anim. Behav. 27, 592; 1979), Gillett and her coworkers offered gregaria desert locusts at a range of densities to a lizard and human predators in an otherwise empty arena. The catching efficiency of the human predators did not vary much, but the effect of increasing prey density on the lizard's catching efficiency was dramatic: above a certain prey density, the lizard became totally incapable of catching any locusts. Gillett et al. attribute these results to a 'visual confusion effect' produced by the activity of the locusts - an effect which, they suggest, would be enhanced by the bold patterning. This remains to be demonstrated, but there are strong indications of the importance of preystriping in other predator - prey interaction (see, for instance, the discussion in Milinski, Z. Tierpsychol., 45, 373; 1977).

Although this is an attractive hypothesis, it may still not be the whole story. There is no reason why the gregaria colour pattern should not have multiple functions, but it is remarkable that neither of the most likely functions in locusts - group cohesion and predator confusion - could be of any use to Australian stick insects: they do not aggregate, and even at high densities, are very inactive.

\section{New human myeloid leukaemia cell line undergoes red shift}

from P.R. Harrison

BIOLOGISTS interested in elucidating how cells acquire their specialised functions (the process of cell differentiation) are always on the look out for ways in which these normal developmental processes can be mimicked by in vitro systems as these often allow the design of more sophisticated experimental approaches than are normally possible in vivo. This applies with even greater force of course to experiments with human cells. For a long time, cells from leukaemic patients or animals have provided useful models for how cells grow and differentiate and the relationship between the occurrence of malignancy and consequent abnormal cell growth and differentiation.

Recently a new human cell line has made something of an impact in the literature on account of its very interesting properties. The K562 cell line was established several years ago by Lozzio and Lozzio working at the University of Tennessee from the pleural effusion of a patient with chronic myeloid leukaemia (CML) in terminal blast crisis (Blood 45, 321; 1975). Initially it was assumed, largely on morphological criteria, that the K562 cells were derived from the outgrowth of a malignant clone of myeloid leukaemic cells arrested at some very early stage of granulocyte

$P$. R. Harrison is at the Beatson Institute for Cancer Research, Glasgow. 